\title{
A rare case of calcifying fibrous pseudotumor of the pleura with an accompanying vascular anomaly in the pulmonary ligament
}

Hanna Lisowska, Marek Marciniak, Jan Cianciara, Konrad Pawełczyk

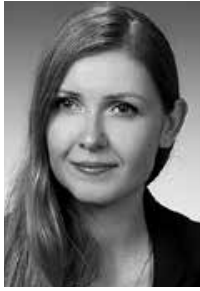

Thoracic Surgery Center, Lower Silesian Centre of Lung Diseases, Wroclaw, Poland

Kardiochirurgia i Torakochirurgia Polska 2018; 15 (1): 59-61

A 27-year old man without any significant comorbidities was admitted to the Department of Thoracic Surgery for the diagnosis and treatment of an operable tumor found accidentally during a routine examination. Tomography revealed a paraspinal tumorous lesion on the left side of the thoracic wall (Figs. 1, 2). The tumor was described as heterogeneous with calcifications in almost $1 / 4$ of its volume. The examination did not show any additional vessels or atypical branches of the main artery.

The presentation of the tumor in magnetic resonance indicated a benign, extrapleural lesion with calcifications, modeling the adjacent lung parenchyma, tangent to the descending aorta and intercostal artery, with no infiltration of the neighboring structures (Fig. 3).

The patient was qualified for tumor resection with the use of video-assisted thoracoscopy. A polycyclic tumorous lesion adjoined to the surface of the lower lobe of the left

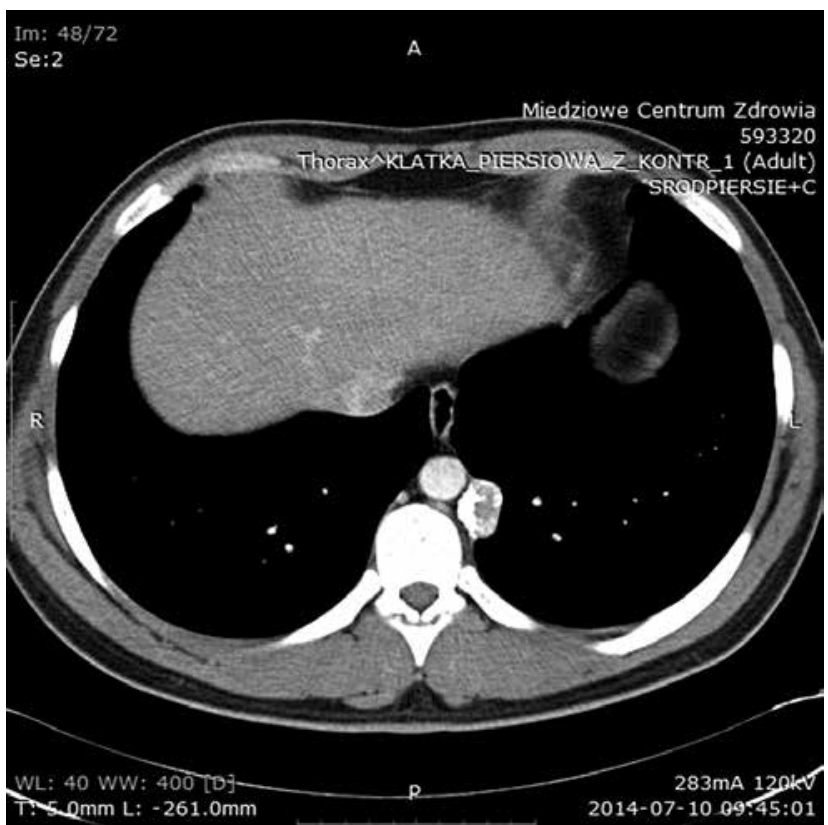

Fig. 1. Tumor visualized by tomographic imaging lung was revealed intraoperatively. Taking into consideration the size and location of the lesion, a decision was made to mobilize the lung by releasing the pulmonary ligament. When a superficial incision of the ligament was performed, an artery was damaged - most likely a branch of the aorta. Conversion to classic anterolateral thoracotomy was performed due to massive hemorrhage. The bloody fluid accumulated in the pleural cavity was evacuated, and pressure was applied to the site of the bleeding. The damaged artery was sewn with vascular sutures on its distal section and the aortic wall. The tumor was excised within normal lung parenchymal margins. Due to significant blood loss, 3 units of packed red blood cells were administered; additional 3 units were administered postoperatively. Other blood derivatives, cryoprecipitate and fresh-frozen plasma (FFP), had to be used as well. A significant decrease in cardiopulmonary parameters necessitated the administration

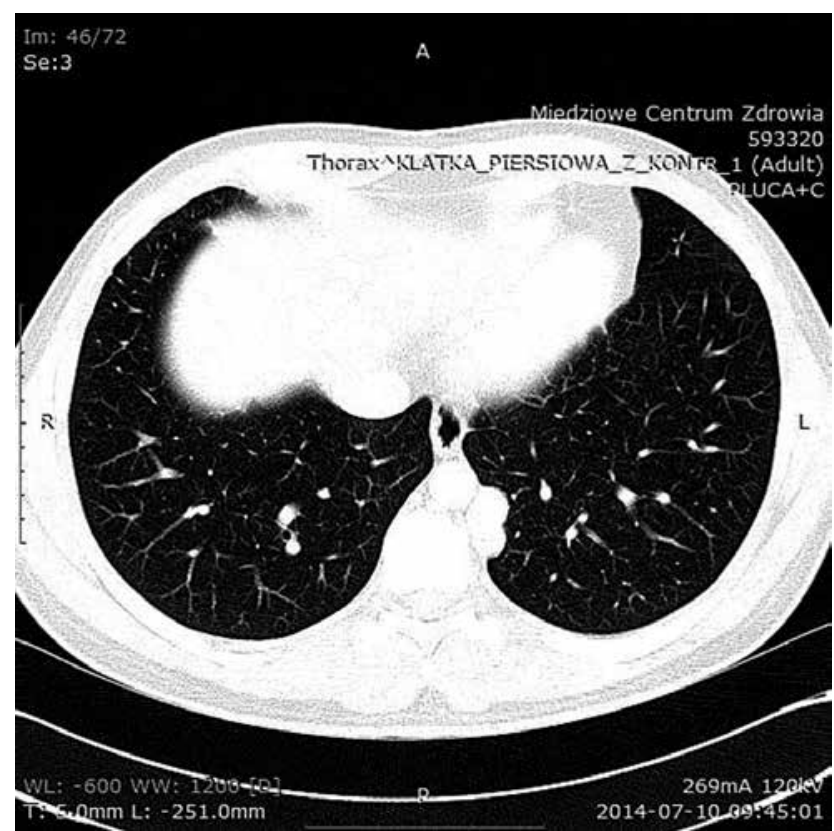

Fig. 2. Tomographic image of the tumor with visible lung parenchyma

Address for correspondence: Hanna Lisowska, Thoracic Surgery Center, Lower Silesian Centre of Lung Diseases, 105 Grabiszyńska St, 53-439 Wroclaw, Poland, phone: +48 7915676 35, e-mail: vea16@interia.pl

Received: 18.01.2017, accepted: 27.07.2017. 


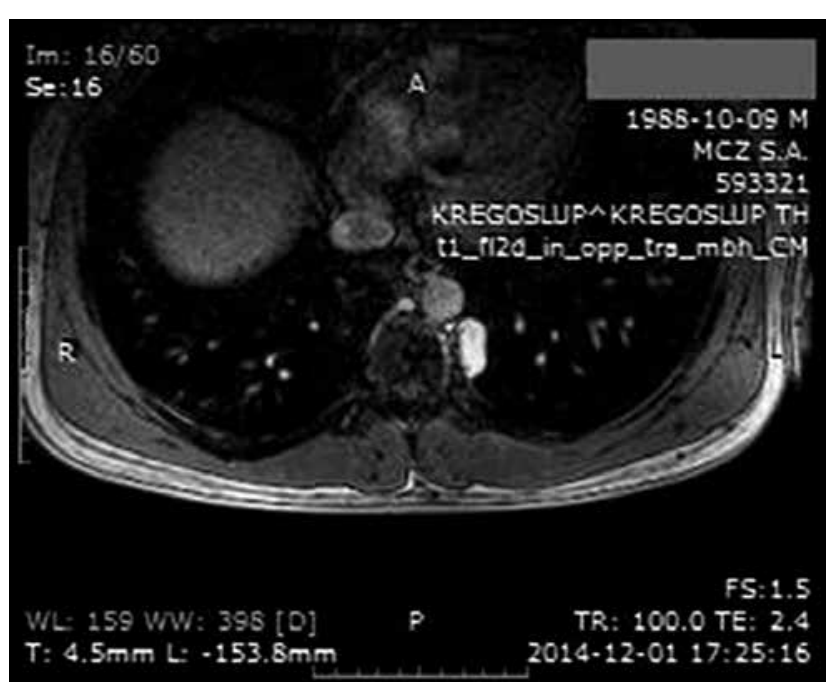

Fig. 3. Magnetic resonance image of the tumor

of catecholamines (even in the postoperative period). The restoration of normal arterial pressure and heart rate increased the drainage dynamics of serous and bloody pleural fluid to over $350 \mathrm{ml} / \mathrm{h}$. Despite the lack of any radiological indications of hematoma, a decision was made to conduct rethoracotomy. The source of bleeding was located in the distal section of the previously sutured artery. Blood leakage was also found on the suture line on the aorta. The sites of bleeding were sewn with vascular sutures and covered with hemostatic dressings. The treatment was continued at the intensive care unit. Cardiopulmonary, morphological, and coagulation parameters were balanced and stabilized (additional 4 units of packed red blood cells and 1 unit of FFP were administered). The drain was removed from the pleural cavity on the first postoperative day. From the third day onwards, the treatment was continued at the Department of Thoracic Surgery. As a result of the catheterization of the subclavian vein, the patient started exhibiting symptoms of thrombophlebitis in the left arm; ultrasound examination confirmed the obstruction of superficial peripheral veins. The patient received antibiotics and therapeutic doses of low-molecular-weight heparin. Ultrasonographic assessment conducted after 3 months revealed complete reperfusion. The patient was discharged in good general condition; he is now monitored by the hospital out-patient clinic. No ailments have been reported so far.

In histopathological assessment, the tumor was characterized as an encapsulated, polycyclic, creamy-white, hypocellular, solid tumorous mass $40 \mathrm{~mm} \times 30 \mathrm{~mm} \times 15 \mathrm{~mm}$ in size; it consisted almost exclusively of collagen fibers and contained psammoma bodies. The lesion was diagnosed as a calcifying fibrous pseudotumor (CFP) of the pleura.

Calcifying fibrous pseudotumor is a tumor rarely encountered in clinical practice, most frequently in children, mainly of the female sex. The first reports come from 1988 - Rosenthal and Abdul-Karim [1] described 2 cases of young patients with atypical lymphoplasmacytic infiltration and calcification of solid lesions. Several years later, in 1993,
Fetsch et al. [2] characterized the histological features of the tumor based on a group of 10 adult patients and proposed the name calcifying fibrous pseudotumor. Microscopic assessment reveals the presence of fusiform cells encircled by a dense collagen matrix with dispersed foci of dystrophic calcification or psammoma bodies and lymphoplasmacytic infiltration. Although instances of multifocal lesions have also been described [3-5], in most cases the tumor is singular and unencapsulated; its growth is usually slow and involves soft tissues. The etiology of the lesion is unknown, but it is classified as a benign mesenchymal tumor [6]. Radical excision of CFPs guarantees low rates of recurrence and secondary focus formation [7].

In the cases described so far, the tumors were most frequently located on the back, scrotum, groin, nape, or armpit; the less common locations included the lungs, pleura, mediastinum, heart, stomach, and mesentery. Pinkard et al. [8] was the first to publish an article devoted to CFPs involving the pleura; since then, 16 similar cases have been described in the available literature [6]. The course of the disease is usually asymptomatic, with discomfort and chest pain being the most frequently reported ailments [9]. Most CFPs originating in the pleura are located in its lower part; pedicles may also be present [6].

Positron emission tomography-computed tomography (PET/CT) examinations do not provide a conclusive diagnosis. The range of fluorine-labeled glucose uptake is estimated at 2.0-4.65. Immunohistochemical examination is not binding: CD 34 is usually negative, (but in the case of lesions originating in the pleura it may be either positive or negative), cytokeratin is usually negative, vimentin positive, and ALK - usually positive. It was suspected that CFPs are late-stage inflammatory myofibrotic tumors (IMT); however, histological comparison and immunohistochemical analysis excluded this possibility [10]. Because of the similarities in histological structure and the positive immunological reaction in the IgG4 class of certain CFP cases, etiological interdependence with lgG4-related diseases (IRD) was also considered. Notwithstanding, differences in location, response to steroids, and recurrence indicate different origins of these diseases [11].

The literature features no reports concerning the coexistence of CFP foci with vascular anomalies. The location of the tumor in the lower lobe of the left lung and the course of the intraoperatively injured artery suggest a similarity to another rare condition - pulmonary sequestration. It is a congenital defect, intra- or extrapulmonary, with independent arterial vascularization originating in the aorta, venous drainage into the lower pulmonary vein in both variants, and, in the extrapulmonary variant, additional drainage into the azygos vein, hemiazygos vein, and inferior vena cava. The disease is usually asymptomatic, with the most common clinical manifestations in the form of recurrent bronchopulmonary inflammations and hemoptysis. In tomographic imaging, intrapulmonary sequestration may present as a cyst cluster or a cyst with fluid or a central cavity; solid masses, nodules, and calcifications are less com- 
mon. In the extrapulmonary variant, it is usually a single, well-defined mass or, less frequently, a cyst [12].

Contrast-enhanced chest tomography constitutes the basis for the diagnostic imaging of lung tumors. In cases of suspected vascular malformations, including pulmonary sequestration, it is also recommended to perform CT angiography, vascular magnetic resonance imaging (MRI), and transesophageal ultrasound with vascular flow imaging.

In the case described in this article, computed tomography and magnetic resonance did not reveal the pathological vascularization in the pulmonary ligament. Since the patient did not report any ailments, the diagnostic imaging performed did not include the assessment of thoracic vascularization.

In cases of arterial hemorrhage, it is necessary to promptly suture the damaged site and resupply the extravasated blood; all the remaining blood factors lost in the bleeding should also be taken into account. The maintenance of hemodynamic parameters is paramount and requires the administration of both catecholamines and infusion fluids. During the early perioperative period, parenteral broadspectrum antibiotic therapy should be applied. Routine use of antibiotics in cases of pulmonary sequestration prevents secondary inflammations of the lungs and bronchi.

\section{Disclosure}

Authors report no conflict of interest.

\section{References}

1. Rosenthal NS, Abdul-Karim FW. Childhood fibrous tumor with psammoma bodies. Clinicopathologic features in two cases. Arch Pathol Lab Med 1988; 112: 798-800

2. Fetsch JF, Montgomery EA, Meis JM. Calcifying fibrous pseudotumor. Am J Surg Pathol 1993; 17: 502-508.

3. Jiang K, Nie J, Wang J, Li J. Multiple calcifying fibrous pseudotumor of the bilateral pleura. Jpn Clin Oncol 2011; 41: 130-133.

4. Azam F, Chatterjee M, Kelly S, Pinto M, Aurangabadkar A, Farooq Latif M, Marshall E. Multifocal calcifying fibrous tumor at six sites in one patient: a case report. World J Surg Oncol 2014; 12: 235.

5. Sleigh KA, Lai W, Keen CE, Berrisford RG. Calcifying fibrous pseudotumours: an unusual case with multiplepleural and mediastinal lesions. Interact Cardiovasc Thorac Surg 2010; 10: 652-3.

6. Lee D, Haam SJ, Choi SE, ParkCH, Kim TH. Calcifying fibrous tumor of the pleura: a rare case with an unusual presentation on CT and MRI. J Korean Soc Radiol 2015; 72: 123-127.

7. Jang KS, Oh YH, Han HX, Chon SH, Chung WS, Park CK, Paik SS. Calcifying fibrous pseudotumor of the pleura. Ann Thorac Surg 2004; 78e: 87-88.

8. Pinkard NB, Wilson RW, Lawless N, Dodd LG, McAdams HP, Koss MN, Travis WD. Calcifying fibrous pseudotumor of pleura. A report of three cases of a newly described entity involving the pleura. Am J Clin Pathol 1996; 105: 189-194.

9. Diniz Ag, Ortaç R, Hoşgör M, Aktaş S, Erbay A. Calcifying fibrous pseudotumor: case report. Ege Tıp Dergisi 2002; 41: 41-42.

10. Hill KA, Gonzalez-Crussi F, Chou PM. Calcifying fibrous pseudotumor versus inflammatory myofibroblastic tumor: a histological and immunohistochemical comparison. Mod Pathol 2001; 14: 784-790.

11. Larson BK, Balzer B, Goldwasser J, Dhall D. Calcifying fibrous tumor: an unrecognized IgG4-related disease? APMIS 2015; 123: 72-76.

12. Serra Salas MM, Castańer González E, Gallardo X, Andreu Magarolas M, Mata Duaso JM, Cano Rodriguez C, Gómez Zaragoza C; Sabadell/ES. Congenital malformations of the pulmonary vessels in adults. Eur Soc Radiol DOI: 10.1594/ecr2016/C-1214. 